\title{
Non-linear and non-local behaviour in spontaneously electrical solids
}

\author{
M. Roman, S. Taj, M. Gutowski, M. R. S. McCoustra, Institute of Chemical Sciences, Heriot-Watt University, \\ Edinburgh, EH14 4AS, UK \\ A. C. Dunn, Institute of Chemical Sciences, Heriot-Watt University, Edinburgh, EH14 4AS, UK \\ Current address: Q ${ }^{2}$ Solutions, The Alba Campus, Rosebank, Livingston, West Lothian, EH54 7EG, UK \\ Z. G. Keolopile, Institute of Chemical Sciences, Heriot-Watt University, Edinburgh, EH14 4AS, UK \\ Current address: Department of Physics, University of Botswana, Private Bag 0022, Gaborone, Botswana \\ Alex Rosu-Finsen, Institute of Chemical Sciences, Heriot-Watt University, Edinburgh, EH14 4AS, UK \\ Current address: Department of Chemistry,University College London, London,WC1H 0AJ, UK \\ A. M. Cassidy, D. Field, Department of Physics and Astronomy, University of Aarhus, Aarhus, DK-8000, Denmark
}

\section{Abstract}

We show that solids displaying spontaneous dipole orientation possess quite general non-local and non-linear characteristics, exemplified through their internal electric fields. The most graphic illustration of this, uncovered originally through electron beam studies, may be found in films of cis-methyl formate (cis-MF). Data demonstrate the counter-intuitive property that the degree of dipole order in the film does not monotonically decrease as the temperature of deposition rises, but rather increases sharply above $77 \mathrm{~K}$. Here we use the independent method of reflectionabsorption infrared spectroscopy (RAIRS) to confirm this result. Our data show that the behaviour of spontelectrics is governed in general, and in particular for cis-MF, by an expression for the degree of dipole orientation, which is continuous in temperature, but with a discontinuity in the derivative. The temperature of deposition associated with this discontinuity matches the temperature above which dipole order switches from the expected reduction with temperature to an increase with temperature. This is believed to be the first example in which the properties of a solid-state system have been found to yield to a continuous mathematical function which shows a set of singularities in the derivative.

When dipolar molecules are laid down from the gas phase, the resulting films can spontaneously possess strong electric fields normal to the plane of the film, whose magnitude can be $>10^{8} \mathrm{Vm}^{-}$ $1,1,2,3,4,5$ Such films are termed 'spontelectric' and are the subject of the present work. The electric field arises through orientation of dipoles within the film, giving rise to a polarization charge on the surface. The extent of dipole orientation is determined by competition between order and disorder and it is therefore natural to assume that films laid down at increasingly higher temperature should show less orientation and therefore a lower electric field. This is indeed observed for numerous species, for example $\mathrm{N}_{2} \mathrm{O}$, toluene, propane, iso-pentane etc. ${ }^{3}$ Using new RAIR spectroscopic data for cis-methyl formate (cis-MF), here we show that the non-linear and non-local characteristics of the spontelectric state can cause the degree of dipole orientation and electric field to increase with increasing temperature of deposition. This strongly reinforces the conclusion based on earlier work, ${ }^{2,3}$ using data from direct measurement of surface polarization potentials, obtained with an electron-beam technique. Figure 1 shows data for the electric field in cis-MF. Our new data underline very clearly the distinctive properties of the spontelectric state of matter.

A mean field model of the spontelectric state has been developed which involves a parametrized electric field in the film of material, in which the net component of the field at any moiety, normal to the plane of the film, $E_{z}$, is written as the sum of a symmetric and an asymmetric part. The symmetric part, related to the 'local field' at any molecular site, ${ }^{6}$ very largely dictates the molecular force field and thus molecular vibrational frequencies in the solid, significant for the present RAIRS data. This part of the field is expressed as a constant term plus a dipole-dipole term, proportional to $\left(\left\langle\mu_{z}\right\rangle / \mu\right)^{2}$, representing the average intermolecular dipole-dipole interactions. ${ }^{7,8,9}$ Here, $\left\langle\mu_{\mathrm{z}}\right\rangle / \mu$, the degree of dipole orientation, is the ratio of the average $\mathrm{z}$-component of the dipole moment and the total dipole moment of the molecular species in the solid state, where the $\mathrm{z}$-axis is normal to the plane of the film. The asymmetric term, arising from the long-range spontelectric field which 
permeates the film, is described by $\left\langle E_{\text {asym }}\right\rangle\left\langle\mu_{\mathrm{z}}\right\rangle / \mu$ and is equal to the observed spontelectric field. The characteristic of the spontelectric state is that $\left\langle\mu_{\mathrm{z}}\right\rangle / \mu$ is non-zero. The polarization or spontelectric field, $\left\langle E_{\mathrm{asym}}\right\rangle\left\langle\mu_{\mathrm{z}}\right\rangle / \mu$, acts in opposition to the symmetrical part of the field and represents the field permeating the medium, created by the average dipoles and experienced by an average dipole.

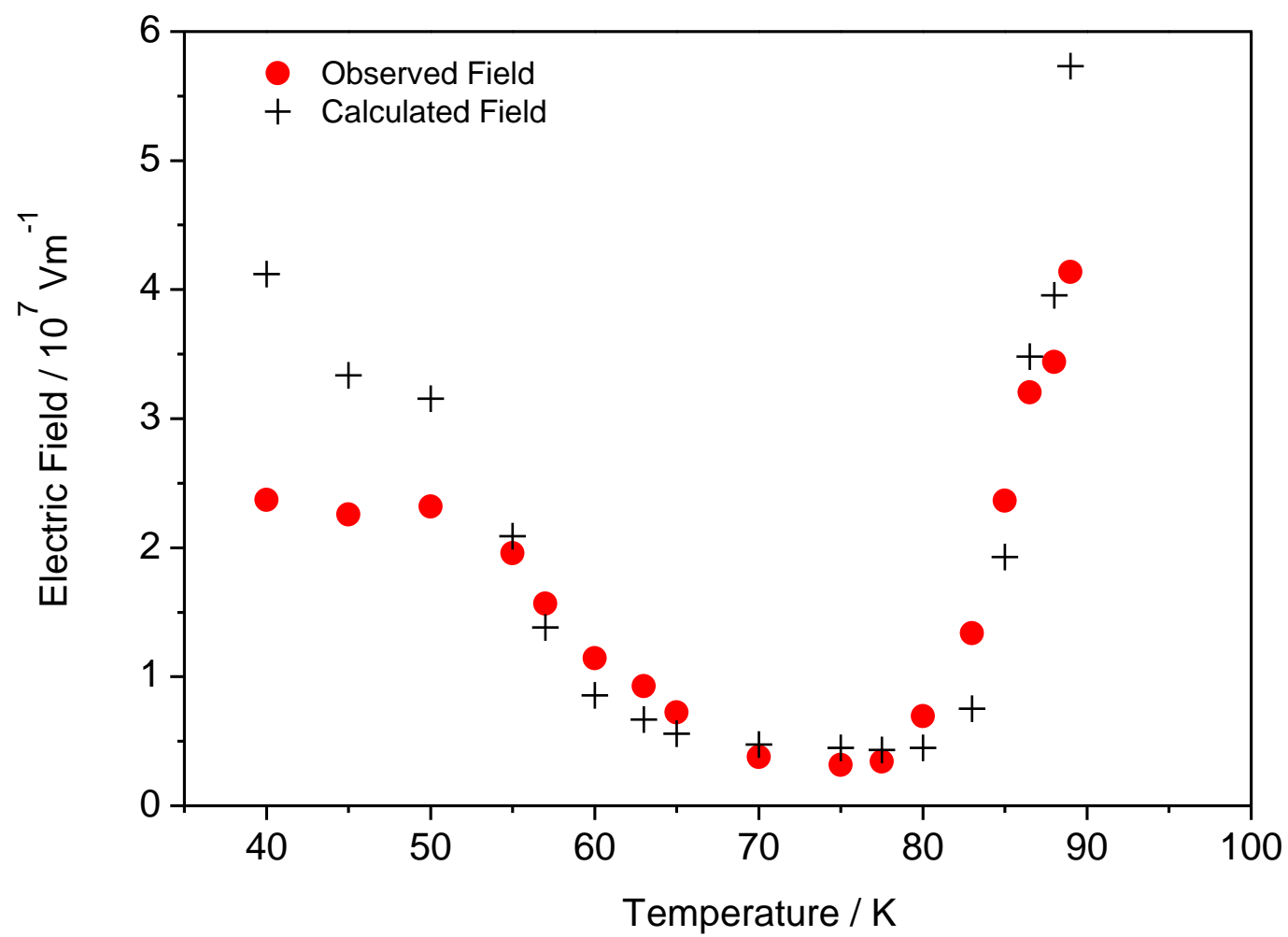

Figure 1: The spontelectric field vs deposition temperature for cis-MF. ${ }^{2,3}$ Calculated values are also shown (see text). Systematic errors due to uncertainties in film thickness may yield errors of up to $30 \%$ in absolute values of electric fields. Random errors in electric fields vary from $1 \%$ at low temperature to $5 \%$ at the highest temperatures.

Hence we may write

$$
E_{\mathrm{z}}=\left\langle E_{\text {sym }}\right\rangle\left[1+\zeta\left(\frac{\left\langle\mu_{\mathrm{z}}\right\rangle}{\mu}\right)^{2}\right]-\left\langle E_{\text {asym }}\right\rangle \frac{\left\langle\mu_{\mathrm{z}}\right\rangle}{\mu}
$$

where $\left\langle E_{\mathrm{sym}}\right\rangle,\left\langle E_{\mathrm{asym}}\right\rangle$ and $\zeta$ are parameters taken to be independent of deposition temperature, over any temperature range for which there is no abrupt structural change. ${ }^{10}$ The $\zeta\left(\left\langle\mu_{\mathrm{z}}\right\rangle / \mu\right)^{2}$ term in equation (1) may be interpreted as a measure of the tendency of one dipolar species to restrict the angular motion of another, a so-called 'frustration' term.

Mean field theory gives an implicit expression for $\left\langle\mu_{\mathrm{z}}\right\rangle / \mu$, yielding the familiar Langevin function for orientational interactions ${ }^{6}$

$$
\frac{<\mu_{\mathrm{z}}>}{\mu}=\operatorname{coth}\left(\frac{E_{\mathrm{z}} \mu}{T}\right)-\left(\frac{E_{\mathrm{z}} \mu}{T}\right)^{-1}
$$


using atomic units, where $T$ is the deposition temperature of the material. Eq. 1 and 2 lead to a total derivative of $\left\langle\mu_{\mathrm{z}}\right\rangle / \mu$ vs $T$, given by

$$
\frac{\mathrm{d}<\mu_{\mathrm{z}} / \mu>}{\mathrm{d} T}=\frac{1 / \mu E_{\mathrm{z}}-\left(\mu E_{\mathrm{z}} / T^{2}\right) \operatorname{cosech}^{2}\left(\mu E_{\mathrm{z}} / T\right)}{\left(\mu E^{\prime} / T\right) \operatorname{cosech}^{2}\left(\mu E_{\mathrm{z}} / T\right)-E^{\prime} T / \mu E_{\mathrm{z}}^{2}-1}
$$

where $E^{\prime}=\left\langle E_{\mathrm{asym}}\right\rangle-2 \zeta\left\langle E_{\mathrm{sym}}\right\rangle\left\langle\mu_{\mathrm{z}}\right\rangle / \mu$. Equation (3) is quite general and has the property that it is continuous in the temperature, but that it can have, for a certain critical range of parameters, a discontinuity in the total derivative with respect to temperature, exemplified by a singular cut in $(T$, $\left\langle\mu_{\mathrm{z}}\right\rangle / \mu$ ) space (figure 27 of [3] for cis-MF). In this connection, cis-MF is presently the only material which we have unequivocally shown to display this singular cut: other materials may of course exist but remain to be found. A general characteristic of equation (3) is a reversal of sign of $\mathrm{d}\left(\left\langle\mu_{\mathrm{z}}\right\rangle / \mu\right) / \mathrm{d} T$ on each side of the cut, being negative on the low temperature and positive on the high temperature side. The positive sign causes the increasing degree of orientation with increasing temperature of deposition, implied by the increasing field at $T \geq 77 \mathrm{~K}$ in Figure 1 . Note that the form of equation 3 arises through the inherent non-locality of the model, in the sense that equations 1 and 2 imply that every part of the film communicates with every other part via the electric field, and through the non-linearity of the model, in the sense that the extent to which the dipoles are oriented, $\left\langle\mu_{\mathrm{z}}\right\rangle / \mu$, depends on the electric field, which itself depends on the degree of orientation.

Turning specifically to methyl formate, electronic structure calculations show that gas phase cis-MF is $19.7 \mathrm{~kJ} \mathrm{~mol}^{-1}$ more stable than trans-MF, at the coupled cluster level of theory ${ }^{11}$ with single and double excitations (CCSD/aug-cc-pVTZ ${ }^{12,13}$ ) in geometry optimizations and frequency calculations, and additional non-iterative triple excitations $(\operatorname{CCSD}(\mathrm{T}))$ in single-point energy calculations. Here the $\operatorname{CCSD}(\mathrm{T})$ energies were extrapolated to the basis set limit. ${ }^{14}$ The value of $19.7 \mathrm{~kJ} \mathrm{~mol}^{-1}$ may be significantly modified through mutual polarization in the solid, but nevertheless indicates a strong preference for cis-MF between $55 \mathrm{~K}$ and $90 \mathrm{~K}$, the temperature range which we consider here (see below). No crystallographic data exist in the literature but IR spectroscopy suggests that solid MF exists in two phases: amorphous and crystalline. The onset of crystallization of MF has been reported for temperatures between 90 and $100 \mathrm{~K}$ in $\operatorname{transmission}^{15}$ and in RAIR spectra ${ }^{16}$, signalled by sharpening of the absorption lines above 90 to $100 \mathrm{~K}$. Mutual polarization in the solid also leads to a strong decrease in the effective dipole moment compared with the gas phase, via $\mu=$ $\mu_{0} /\left(1+\alpha k / s^{3}\right)$, where $s$ is the average spacing between successive layers, $\alpha$ is the molecular polarizability, $k=11.034$ and $\mu_{0}$ is the gas phase dipole moment of the molecules involved. For cisMF, $\mathrm{s}=0.244 \mathrm{~nm}, \alpha=5.25 \times 10^{-30} \mathrm{~m}^{3}, \mu_{0}=0.6949 \mathrm{au}$ (or $1.766 \mathrm{D}$ ), giving $\mu=0.354 \mathrm{D}^{2,3}$

Fitting data for deposition temperatures between 55 and $75 \mathrm{~K}$ to equations (1) and (2), yields $\left\langle E_{\text {sym }}\right\rangle$ $=1.206 \times 10^{7} \mathrm{~V} \mathrm{~m}^{-1},\left\langle E_{\text {asym }}\right\rangle=1.148 \times 10^{9} \mathrm{~V} \mathrm{~m}^{-1}, \zeta=1.45 \times 10^{4}$. These values then go on to predict the observed qualitatively distinctive rise in the spontelectric field for deposition temperatures above $75 \mathrm{~K}$, as seen in Figure 1 and in Table 1 for $\left\langle\mu_{\mathrm{z}}\right\rangle / \mu$. Below $55 \mathrm{~K}$ the model fails, most likely due to involvement of a different phase of cis-MF at lower temperatures. We therefore limit discussion to temperatures of deposition $\geq 55 \mathrm{~K}$ and focus upon the anomalous behaviour of cis-MF above $\sim 75 \mathrm{~K}$. We now present RAIRS data for $c i s-\mathrm{MF}$ for $55<\mathrm{T}<90 \mathrm{~K}$ and show that these new data corroborate the counter-intuitive behaviour of the spontelectric field (Figure 1) and the degree of dipole orientation (Table 1).

The use of RAIRS to measure the properties of spontelectric solids is based on the vibrational Stark effect, via the response of molecular vibrations to an electric field in the film. The technique has 
been validated ${ }^{17}$ for $\mathrm{N}_{2} \mathrm{O}$ films, through comparison with values of surface potentials and corresponding spontelectric fields obtained using the electron beam technique. In addition, RAIRS studies of the spontelectric effect in solid CO yield spontelectric fields ${ }^{18}$ which match those which reproduce spectral shifts in VUV spectra of solid CO. ${ }^{5}$ RAIRS spectra of cis-MF films, grown by vapour deposition at various temperatures, were obtained at Heriot-Watt University, using a Fourier-transform infrared spectrometer (Varian 670-IR) in reflection-absorption mode, at a grazing incidence of $75^{\circ}$ with respect to the normal to the substrate. The substrate was oxygen-free, high conductivity copper, coated with a $300 \mathrm{~nm}$ layer of amorphous silica, whose presence allows the detection of TO modes. ${ }^{17}$ Spectra, taken at a resolution of $0.1 \mathrm{~cm}^{-1}$, thus show LO-TO splitting, key for the analysis involving the spontelectric effect. Samples were deposited at a range of temperatures but were cooled to $18 \mathrm{~K}$ before spectra were recorded, noting that this introduced no distortion into our data, since changes in dipole orientation are not temperature reversible. ${ }^{3}$ Errors in temperature measurement, performed using a KP-type thermocouple, were $\pm 0.5 \mathrm{~K}$.
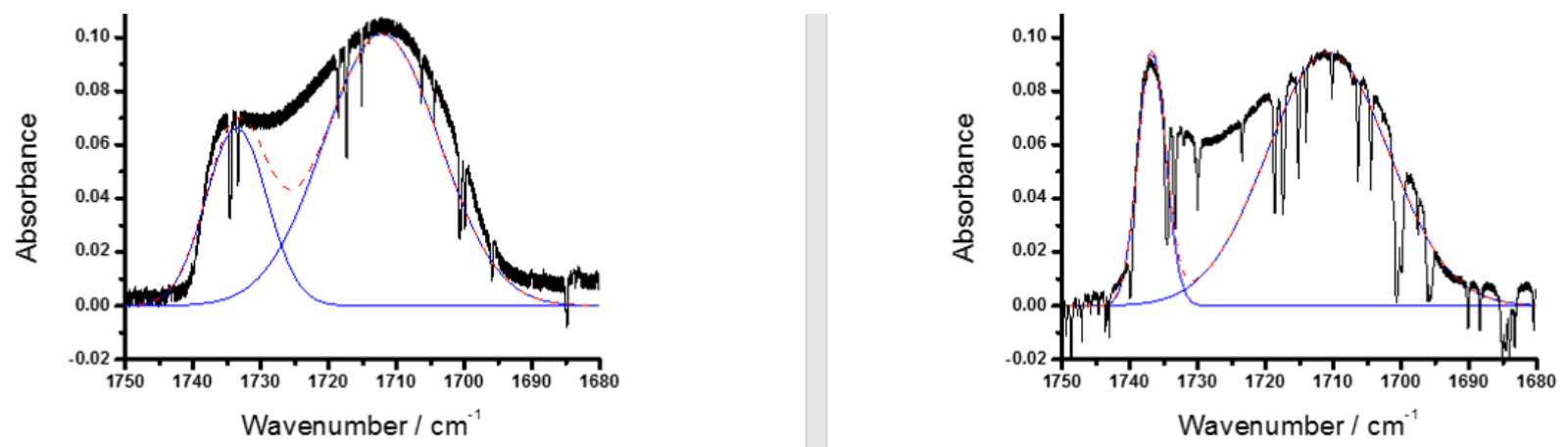

Figure 2: RAIR spectra of solid cis-methyl formate deposited on amorphous silica film on a copper surface (black lines). Estimates based on coverage dependence of TPD places the film thickness at a few 10's of ML. The sharp spectral features are due to residual water vapour in the purge gas of our external optics, where features are useful as wavelength markers. Spectra on the lhs were recorded at $70 \mathrm{~K}$ and on the rhs at $90 \mathrm{~K}$ deposition temperatures and were obtained by co-adding 512 scans at a resolution of $0.1 \mathrm{~cm}^{-1}$ in typically 60 minutes. The blue lines are Gaussian components representing the LO and TO modes in the solid obtained by nonlinear fitting using Origin. LO-TO splittings are given in Table 1. The red dashed line shows the sum of the fits, the greater discrepancy at $90 \mathrm{~K}$ compared with $70 \mathrm{~K}$ indicating increased disordered molecular structure at higher deposition temperature.

\begin{tabular}{|c|c|c|c|c|}
\hline $\begin{array}{c}\text { Deposition } \\
\text { Temperature / } \mathrm{K}\end{array}$ & $\mathrm{LO} / \mathrm{cm}^{-1}\left(v_{\mathrm{L}}\right)$ & $\mathrm{TO} / \mathrm{cm}^{-1}\left(v_{\mathrm{T}}\right)$ & $\begin{array}{c}\text { Splitting } \Delta v \\
/ \mathrm{cm}^{-1}\end{array}$ & $\begin{array}{c}\text { Degree of dipole } \\
\text { orientation }\end{array}$ \\
\hline 55 & & & & \\
\hline 60 & $1733.8 \pm 0.4$ & $1713.2 \pm 1.6$ & $20.6 \pm 1.6$ & 0.0152 \\
\hline 65 & $1733.6 \pm 0.4$ & $1712.8 \pm 1.0$ & $20.8 \pm 1.1$ & 0.0089 \\
\hline 70 & $1733.2 \pm 0.8$ & $1712.0 \pm 0.8$ & $21.2 \pm 1.1$ & 0.0056 \\
\hline 75 & $1722.7 \pm 0.6$ & $1712.0 \pm 0.2$ & $21.7 \pm 0.6$ & 0.0029 \\
\hline 80 & $1734.8 \pm 0.4$ & $1711.6 \pm 0.2$ & $23.2 \pm 0.4$ & 0.0025 \\
\hline 85 & $1735.6 \pm 0.6$ & $1711.6 \pm 0.6$ & $24.0 \pm 0.8$ & 0.0054 \\
\hline 90 & $1736.4 \pm 0.2$ & $1711.4 \pm 0.2$ & $25.0 \pm 0.3$ & 0.0184 \\
\hline & $1736.8 \pm 0.2$ & $1710.8 \pm 0.4$ & $26.0 \pm 0.4$ & 0.0322 \\
\hline
\end{tabular}

Table 1. Column 1: deposition temperature of cis-MF film. Column 2: Longitudinal oscillation (LO) frequency of the CO stretch. Column 3: Transverse oscillation (TO) frequency of the CO stretch. Column 4: measured LO-TO splitting. Column 5: degree of dipole orientation, $\left\langle\mu_{z}\right\rangle / \mu$, from earlier work. ${ }^{2,3}$

From hereon we consider only the readily identifiable $\mathrm{C}=\mathrm{O}$ stretching mode and associated LO-TO splitting, which lies between 1733.8 and $1736.8 \mathrm{~cm}^{-1}$ for LO and between 1710.8 and $1713.2 \mathrm{~cm}^{-1}$ for $\mathrm{TO}$ modes. Two typical spectra, at $70 \mathrm{~K}$ and $90 \mathrm{~K}$, focussing on this $\mathrm{C}=\mathrm{O}$ region in cis-MF, are 
shown in Figure 2. Table 1 demonstrates that the LO-TO splitting rises markedly between 55 and $90 \mathrm{~K}$. The increase in splitting at higher deposition temperatures in Table 1 is sufficient to illustrate a clear general trend of an increasing vibrational Stark effect. Thus RAIRS data immediately provide a qualitative corroboration of the anomalous deposition temperature dependence of both the spontelectric field and the degree of dipole orientation in cis-MF. We now seek to put this on a quantitative footing.

The LO-TO splittings, $v_{\mathrm{L}}-v_{\mathrm{T}}=\Delta v$, in Table 1 arise through a combination of the intrinsically different vibrational frequencies associated with LO and TO modes and, at the level of approximation adopted here, an independent contribution due to the vibrational Stark effect. Thus the observed values of $\Delta v$ are the sum of $\Delta v_{\mathrm{B}}$, the intrinsic splitting, essentially independent of temperature of deposition, and $\Delta v_{\mathrm{S}}$, the spontelectric Stark term, which is a function of temperature through the temperature dependence of the spontelectric field. The intrinsic splitting is determined largely by the size of the unit cell. ${ }^{19}$ Initially we assume that this size remains approximately constant with temperature, irrespective of phase change. $\Delta v_{\mathrm{B}}$ can then be estimated from spectra of the crystalline phase formed by deposition above $90 \mathrm{~K}$, at which temperatures the solid is no longer spontelectric and there are therefore no spontelectric contributions to the LO-TO splitting, yielding $\Delta v_{\mathrm{B}}=13.5 \mathrm{~cm}^{-1}$.

It has been shown ${ }^{17}$ that the difference in energy between TO and LO vibrations divided by the TO vibrational energy, that is, $\left(U_{\mathrm{T}}-U_{\mathrm{L}}\right) / U_{\mathrm{T}}, \sim \Delta v / v_{\mathrm{T}} . U_{\mathrm{T}}$ and $U_{\mathrm{L}}$ are related to quantities governing the spontelectric effect, $\left\langle E_{\mathrm{sym}}\right\rangle,\left\langle E_{\mathrm{asym}}\right\rangle, \zeta$ and $\left\langle\mu_{\mathrm{z}}\right\rangle / \mu$, since these define the environment of the constituent molecules. First, the total field governing $\nu_{\mathrm{T}}$ (say) must be proportional to $\left(\Delta v / \Delta v_{\mathrm{S}}\right)<E_{\mathrm{sym}}>\left(1+\zeta\left(\left\langle\mu_{\mathrm{Z}}>/ \mu\right)^{2}\right)\right.$, where $\Delta v / \Delta v_{\mathrm{S}}$ is equal to the ratio of the total splitting to that caused by the spontelectric field, and second, $U_{\mathrm{T}}-U_{\mathrm{L}} \propto E_{\mathrm{S}}\left\langle\mu_{\mathrm{z}}\right\rangle / \mu$, the projection of the spontelectric field, $E_{S}$, onto the direction in which the average dipole points. This yields

$$
\frac{\Delta v}{v_{\mathrm{T}}} \approx \frac{\left(\Delta v_{\mathrm{S}} / \Delta v\right)\left\langle E_{\text {asym }}\right\rangle\left(\left\langle\mu_{\mathrm{z}}\right\rangle / \mu\right)^{2}}{\left\langle E_{\mathrm{sym}}\right\rangle\left[\left(1+\varsigma\left(\left\langle\mu_{\mathrm{z}}\right\rangle / \mu\right)^{2}\right)\right]}+\frac{\Delta v_{\mathrm{B}}}{v_{\mathrm{T}}}
$$

Using the empirical observation that $\mathrm{d} \nu_{\mathrm{T}} / \mathrm{d}\left(\left\langle\mu_{\mathrm{Z}}\right\rangle / \mu\right) \sim-\mathrm{d} \nu_{\mathrm{L}} / \mathrm{d}\left(\left\langle\mu_{\mathrm{Z}}\right\rangle / \mu\right)$, the differential of (4) with respect to $\left\langle\mu_{\mathrm{z}}\right\rangle / \mu$ gives:

$$
\begin{aligned}
& \frac{\mathrm{d} \nu_{\mathrm{T}}}{\mathrm{d}\left\langle\mu_{\mathrm{z}}\right\rangle / \mu}= \\
& -\frac{\left\langle E_{\text {sym }}\right\rangle\left\langle E_{\text {asym }}\right\rangle\left(\left\langle\mu_{\mathrm{z}}\right\rangle / \mu\right)\left(v_{\mathrm{L}}-\Delta v_{\mathrm{B}}\right)\left\{2 \xi+\left(\left\langle\mu_{\mathrm{z}}\right\rangle / \mu\right) \eta \xi^{\prime}\right\}}{\left.\left.\left\{\left\langle E_{\text {sym }}\right\rangle \eta+\left\langle E_{\text {asym }}\right\rangle\left(\left\langle\mu_{\mathrm{z}}\right\rangle / \mu\right)^{2} \xi\right]\right\}\left\{2\left\langle E_{\text {sym }}\right\rangle \eta+\left\langle E_{\text {asym }}\right\rangle\left(\left\langle\mu_{\mathrm{z}}\right\rangle / \mu\right)^{2} \xi\right]\right\}}
\end{aligned}
$$

where $\xi=\Delta v_{\mathrm{S}} / \Delta v, \eta=1+\zeta\left(\left\langle\mu_{\mathrm{z}}\right\rangle / \mu\right)^{2}$ and $\xi^{\prime}=\mathrm{d}\left(\Delta v_{\mathrm{S}} / \Delta v\right) / \mathrm{d}\left(\left\langle\mu_{\mathrm{z}}\right\rangle / \mu\right)$. Forming the product of eq. 5 with $\mathrm{d}\left(\left\langle\mu_{\mathrm{z}}\right\rangle / \mu\right) / \mathrm{d} T$ in eq. 3 gives $\mathrm{d} \nu_{\mathrm{T}} / \mathrm{d} T$. Using the approximation $\mathrm{d} \nu_{\mathrm{T}} / \mathrm{d} T=-\mathrm{d} \nu_{\mathrm{L}} / \mathrm{d} T$, this yields on suitable numerical integration the value of $\Delta v$ versus $T$, as desired.

Before this can be accomplished, some value of $\xi^{\prime}$ must however be adopted. This was conveniently achieved by a simple fitting procedure based on the observed total LO-TO splitting, 
values of $\Delta v_{\mathrm{B}}$ and corresponding values of $\left\langle\mu_{\mathrm{z}}\right\rangle / \mu$ (see Table 1). For $\Delta v_{\mathrm{B}}=13.5 \mathrm{~cm}^{-1}$, this gives $\xi^{\prime}$ $=0.399-8.02\left\langle\mu_{\mathrm{z}}>/ \mu+295\left(\left\langle\mu_{\mathrm{z}}\right\rangle / \mu\right)^{2}\right.$ for the range $T_{\mathrm{dep}}=55,60,65$ and $70 \mathrm{~K}$ and $\xi^{\prime}=0.399+$ $\left.9.66<\mu_{\mathrm{z}}\right\rangle / \mu-503\left(\left\langle\mu_{\mathrm{z}}\right\rangle / \mu\right)^{2}+8.85 \times 10^{3}\left(\left\langle\mu_{\mathrm{z}}\right\rangle / \mu\right)^{3}$ for $T=75,80,85$ and $90 \mathrm{~K}$ with very similar results for example with $\Delta v_{\mathrm{B}}=10 \mathrm{~cm}^{-1}$ (see below).

What remains is to insert values of the spontelectric parameters into the product of equations 3 and 5 , represent the resulting derivatives $\mathrm{d} v_{\mathrm{T}} / d T$ or $\mathrm{d} v_{\mathrm{L}} / d T$ as a polynomial in $T$ and integrate the result. This yields the variation of the LO-TO splitting, $\Delta v$, versus deposition temperature, shown in Fig. 3 obtained using the appropriate parameters for $c i s-\mathrm{MF}^{2,3}$ already provided. Fig. 3 shows a satisfactory agreement between model and observation. We stress that this agreement is the result of the use of analytical forms with proscribed experimental parameters and does not represent a 'fit' to experimental data.

The greatest source of numerical uncertainty in the calculated values of the LO-TO splitting, shown in Figure 3 , is the uncertainty in the value of $\Delta \nu_{\mathrm{B}}$, the inherent LO-TO splitting. $\Delta v_{\mathrm{B}}$ may assume a different value from the average observed value for $>90 \mathrm{~K}$ of $\sim 13.5 \mathrm{~cm}^{-1}$ used above, since $\Delta v_{\mathrm{B}}$ is structure dependent and MF crystallizes at $>90 \mathrm{~K} .{ }^{15,16}$ The dependence of the LO-TO splitting on $\Delta \nu_{\mathrm{B}}$ analytically contains the total derivative governing the variation of the degree of dipole orientation with temperature, eq. 3. Any choice of $\Delta v_{\mathrm{B}}$ which brings us close to associated singularities yields numerically unreliable results for the LO-TO splitting and thus one may not freely vary the value of $\Delta v_{\mathrm{B}}$. With this in mind, an uncertainty of, say, $3.5 \mathrm{~cm}^{-1}$ in $\Delta v_{\mathrm{B}}$ yields an uncertainty in the calculated LO-TO splitting of $\leq 0.5 \mathrm{~cm}^{-1}$. In order to illustrate this, two estimates of the LO-TO splitting are shown in Figure 3, for $\Delta v_{\mathrm{B}}=10$ and $13.5 \mathrm{~cm}^{-1}$. Note that a lower value of $\Delta v_{\mathrm{B}}$ is consistent with a larger unit cell in amorphous as opposed to crystalline MF.

The significance of the current work is that it underpins the non-linear and non-local nature of the spontelectric effect, as described by equations 1 and 2 . These equations predict a perceived increase in dipole ordering, represented by $\left\langle\mu_{\mathrm{z}}\right\rangle / \mu$, with increasing deposition temperature, for a certain range of values of the spontelectric parameters, $\left\langle E_{\mathrm{sym}}\right\rangle,\left\langle E_{\mathrm{asym}}\right\rangle$ and $\zeta$. The goal here was to provide independent data for the remarkable phenomenon of the growth of dipole order with increasing temperature .2,3 Results in figure 3 show that the parameters which define the spontelectric effect in cis-MF, obtained in earlier work based on the e-beam technique and derived using equations 1 and 2, can be used to reproduce the observed temperature dependence of LO-TO splitting measured in RAIR spectroscopy of cis-MF. This provides the independent evidence which we seek.

This study is an illustration of how the mathematical structure of a model can lead to new physics. The nature of the spontelectric state suggests a description in terms of an electric field and the Langevin equation, yielding a model which is successful in reproducing the variation of the spontelectric field with deposition temperature for a variety of materials, such as $\mathrm{N}_{2} \mathrm{O}$ or the three chlorofluorocarbons $\mathrm{CF}_{3} \mathrm{Cl}, \mathrm{CF}_{2} \mathrm{Cl}_{2}, \mathrm{CFCl}_{3}{ }^{3}$. This description then throws up unusual mathematical properties which turn out to reproduce the observation of quite unexpected physical behaviour in cis-MF. This behaviour was not used as a basis for the model: indeed it was quite unknown when the model was put forward. In this sense the mathematical analysis has become the vehicle through which new physics was explored 


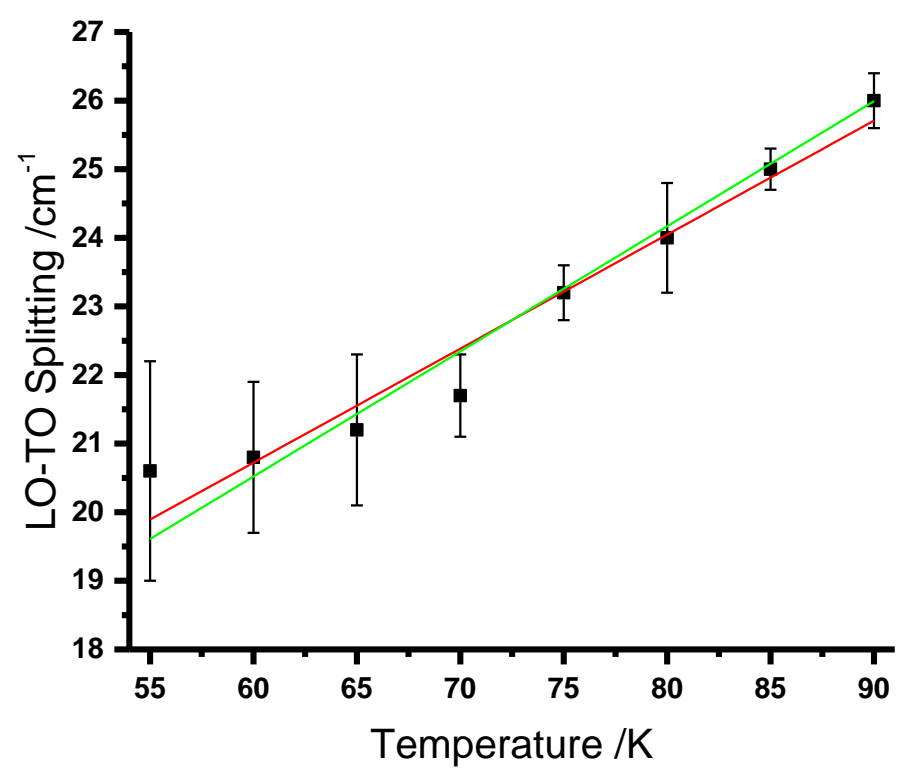

Fig. 3 Comparison between the model (green and red lines) and the observed LO-TO splitting (black squares) with respect to deposition temperature. Two sets of results are shown for $\Delta v_{\mathrm{B}}=10$ and $13.5 \mathrm{~cm}^{-1}$, green and red respectively. Uncertainties in the temperature are of the order of the size of the symbols in the figure, that is, $\pm 0.25 \mathrm{~K}$. For a discussion of the uncertainties shown in the calculated values of the LO-TO splitting, see text.

Present data illustrate that spontelectrics provide a nanoscopic example of a realisable state of matter, whose properties are described by a continuous function, which is non-differentiable at a set of points in parameter space and in which the state of a system can show discontinuity in response to a continuous influence. Results in earlier work were sufficiently puzzling that we sought and have presented independent experimental evidence which places our description of the spontelectric state upon a very sound basis. With regard to a qualitative understanding of what is afoot here, species as they attach from the gas phase to the surface evidently find it favourable at all temperatures to form a dipole-oriented structure. The formation of such structures is enhanced by weakly hindered diffusion and less restriction to rotation of the molecular constituents. Greater thermal diffusion in higher temperature films implies that species are able to explore a greater volume of phase space, overcoming barriers of frustration imposed by $\left\langle\mathrm{E}_{\mathrm{sym}}\right\rangle$ and $\zeta$ between metastable states. Less frustrated rotation concurrently may allow molecules more readily to align and more than offset the increased disorderly motion associated with higher temperature. This in turn increases the degree of dipole alignment that can be achieved in the system at higher deposition temperatures. This description calls for verification with a dynamical model which describes how the spontelectric state may be established and maintained.

MM wishes to acknowledge the supported of the U.K. Science and Technology Facilities Council (STFC, ST/M001075/1), the U.K. Engineering and Physical Sciences Research Council (EPSRC, GR/T27044/02) and the European Community FP7-ITN and H2020-ITN Marie Curie Programmes (LASSIE Project, Grant Agreement 238258; and EuroPAH Project, Grant Agreement 722346).

\section{References}

\footnotetext{
${ }^{1}$ R. Balog, P. Cicman, N. Jones, and D. Field, Phys. Rev. Lett., 2009, 102, 2-5.
} 
${ }^{2}$ O. Plekan, A. Cassidy, R. Balog, N. C. Jones, and D. Field, Phys. Chem. Chem. Phys., 2012, 14, 9972-6.

${ }^{3}$ D. Field, O. Plekan, A. Cassidy, R. Balog, N. C. Jones, and J. Dunger, Int. Rev. Phys. Chem., 2013, 32, 345-392

${ }^{4}$ Oksana Plekan, Alexander Rosu-Finsen, Andrew M. Cassidy, Jerome Lasne, Martin R.S. McCoustra and David Field, Eur. Phys. J. D (2017) 71: 162

${ }^{5}$ Y.-J. Chen, G. M. Muñoz Caro, S. Aparicio, A. Jiménez-Escobar, J. Lasne, A. Rosu-Finsen, M. R.S. McCoustra, A.

M. Cassidy, D. Field. Phys.Rev.Lett. 2017, 119, 157703

${ }^{6}$ C. Kittel, Introduction to Solid State Physics, Wiley, 3rd edn., 2005.

${ }^{7}$ E. Cohen de Lara and J. Vincent-Geisse, J. Phys. Chem., 1976, 80, 1922

${ }^{8}$ B. L. Maschhoff and J. P. Cowin, J. Chem. Phys., 1994, 101, 8138.

${ }_{9}^{9}$ D. Fernández-Torre, O. Kupiainen, P. Pyykkö, and L. Halonen, Chem. Phys. Lett., 2009, 471, 239

${ }^{10}$ A. Cassidy, M. R. V. Jørgensen, A. Rosu-Finsen, J. Lasne, J. H. Jørgensen, A. Glavic, V. Lauter, B. B. Iversen, M. R.S. McCoustra and D. Field. J. Phys. Chem. C, 2016, 120, 24130

${ }^{11}$ R. J. Bartlett and M. Musiał, Rev. Mod. Phys. 2007, 79, 291-352.

${ }^{12}$ T. H. Dunning, J. Chem. Phys. 1989, 90, 1007-1023.

${ }^{13}$ R. A. Kendall, T. H. Dunning, R. J. Harrison, J. Chem. Phys. 1992, 96, 6796-6806.

${ }^{14}$ R. A. Bachorz, W. Klopper, M. Gutowski, J. Chem. Phys. 2007, 126, 085101.

${ }^{15}$ P. Modica and M. E. Palumbo, Astron. Astrophys., 2010, 519, A22

${ }^{16}$ D. J. Burke, F. Puletti, P. M. Woods, S. Viti, B. Slater and W. A. Brown, J. Phys. Chem. A, 2015, 119, 6837

${ }^{17}$ J. Lasne, A.Rosu-Finsen, A.Cassidy, M.R.S.McCoustra, D. Field, Phys. Chem. Chem. Phys., 2015, 17, 20971

${ }_{18}^{18}$ Rosu-Finsen, A., Lasne, J, Cassidy, A., McCoustra M.R.S., Field, D.,Phys.Chem.Chem.Phys. 18, 5159-5171, (2016)

${ }^{19}$ L.H. Jones, B.I. Swanson, J. Phys. Chem., 1991, 95, 2701 